\title{
Empleo del paquete ExpRep para repetición de ensayos de Bernoulli en la enseñanza de las probabilidades
}

\author{
Larisa Zamora \\ larisa@uo.edu.cu \\ Universidad de Oriente. \\ Santiago de Cuba. Cuba
}

\author{
Jorge Díaz \\ jdiaz@uo.edu.cu \\ Universidad de Oriente. \\ Santiago de Cuba. Cuba
}

\begin{abstract}
El paquete ExpRep permite calcular probabilidades de ocurrencia de un determinado evento en un gran número de repeticiones de un ensayo de tipo Bernoulli, mediante la aplicación de los teoremas local e integral de De Moivre-Laplace y el teorema de Poisson, dando la posibilidad de mostrar gráfica y analíticamente los resultados en aras de compararlos con aquellos alcanzados a partir de la aplicación directa de la fórmula binomial. Se presentan además tres aplicaciones del teorema integral de De Moivre-Laplace y la simulación del experimento de Buffon para cuatro valores diferentes del número de repeticiones del ensayo. El paquete fue desarrollado en el lenguaje de programación $\mathrm{R}$ y responde fundamentalmente a objetivos didácticos de la enseñanza de las Probabilidades para el nivel universitario de pregrado en Matemática.
\end{abstract}

Palabras clave: Repetición de ensayos, ensayo de Bernoulli, teorema de Poisson, teoremas de De Moivre-Laplace.

\begin{abstract}
The package ExpRepallows to calculate probabilities of occurrences of a given event in a large number of repetitions of a Bernoulli trial, by applying the local and integral theorems of De MoivreLaplace and the Poisson theorem, giving the possibility of displaying graphically and analytically the results in order to compare them with those achieved from the direct application of the binomial formula. Additionally, three applications of the integral theorem of De Moivre-Laplace and the simulation of the Buffon experiment for four different values of the number of repetitionsare presented.The package was developed in the $\mathrm{R}$ programming language, and it is useful for teaching Probability in undergraduate courses in Mathematics.
\end{abstract}

KeyWords: Experiment repetition, Bernoulli trial, Poisson theorem, theorems of De Moivre-Laplace 


\subsection{Introducción}

Por lo general, el proceso de la enseñanza de la Probabilidad comienza con la presentación de conceptos que previamente ha preparado el profesor junto con ejemplos, el uso de algún material manipulativo y ocasionalmente el computador; todo con el interés de que el estudiante se apropie del tema y haga posteriormente su aplicación en un contexto. El uso de las TIC (Tecnologías de la Información y las Comunicaciones) en la educación ha contribuido a la generación de nuevas estrategias en el proceso de enseñanza y aprendizaje, por lo que el docente necesita buscar nuevos métodos que capten la atención de los estudiantes y hagan más efectiva la enseñanza y el aprendizaje de la Probabilidad en el contexto de la educación superior (Osorio, [9, (2013)]).

Barrera y Santos (citados por Gamboa, 2007) consideran que el uso de la tecnología puede llegar a ser una poderosa herramienta para que los estudiantes logren crear diferentes representaciones de ciertas tareas y sirve como un medio para que formulen sus propias preguntas o problemas, lo que constituye un importante aspecto en el aprendizaje de las matemáticas.

Según Olesker [8, (2015)], con el desarrollo de la informática ha aumentado el interés por la introducción experimental de la probabilidad comolímite de frecuencia estabilizada. A partir de las simulaciones los estudiantes podrían superar algunas de las paradojas que surgen en problemas sencillos, así como enfrentarse a ideas erróneas que frecuentemente tienen sobre el azar y sus características. Más adelante este autor plantea: "Se puede afirmar que el uso de programas de simulación permite poner en manos del alumno un nuevo instrumento que hace posible la exploración y el descubrimiento de conceptos y principios que de otro modo serían mucho más abstractos, contribuyendo a solucionar el problema de la falta de experiencia estocástica y a la mejora de la intuición probabilística que, en general, no se desarrolla espontáneamente" (p.90).

El trabajo que se presenta va encaminado en esta dirección, o sea, emplear la computación para ayudar a la comprensión de los estudiantes en los tópicos de la teoría de las probabilidades que se detallan a continuación, con el auxilio de un paquete elaborado por los autores del presente trabajo en el lenguaje de programación R (Matloff, [7, (2011)]), versión 3.3.0, el cual permite:

- Calcular la probabilidad de que un evento $A$ ocurra en una cantidad dada de repeticiones independientes de un ensayo de Bernoulli, de forma exacta y aproximada mediante la aplicación de los teoremas local e integral de De Moivre-Laplace y el de Poisson, proporcionando salidas gráficas y numéricas.

- Calcular el número n de repeticiones del ensayo de Bernoulli, para que la probabilidad de que la frecuencia de ocurrencia del evento se desvíe de su verdadera probabilidad $p(p=P(A))$ en valor absoluto una cantidad no mayor que $\alpha$, sea igual a $\beta$, esto es, calcular $n$ tal que:

$$
P\left(\left|\frac{m}{n}-p\right|<\alpha\right)=\beta .
$$

- Calcular el valor de $\alpha$ dado los valores de $n$ y $\beta$ de forma tal que se verifique la ecuación (1.1).

- Calcular el valor de $\beta$ dado los valores de $n$ y $\alpha$ de forma tal que se verifiquela ecuación (1.1). 
Estos tres últimos cálculos responden a aplicaciones del teorema integral de De Moivre-Laplace.

- Simular el experimento de Buffon para cuatro valores diferentes del número de repeticiones.

\subsection{Bases teóricas}

A continuación se brindan algunas definiciones y teoremas concernientes a la repetición de experimentos independientes de tipo Bernoulli, que constituyen la base teórica del paquete.

\section{Definición 1.1}

Un ensayo de Bernoulli es un experimento aleatorio en el cual sólo pueden ocurrir dos resultados: el éxito con probabilidad $p$ y el fallo con $\operatorname{probabilidad} q=1-p$.

\section{Definición 1.2}

Un experimento de Bernoulli es el que resulta de la repetición en el tiempo de ensayos de Bernoulli independientes y para cada ensayo la probabilidad $p$ de aparición del evento éxito es constante.

Usualmente existe el interés en calcular la probabilidad $P_{n}(m)$ de que el evento éxito ocurra exactamente $m$ veces en $n$ repeticiones de un ensayo de Bernoulli, para lo cual se emplea la distribución binomial, esto es,

$$
P_{n}(m)=\left(\begin{array}{c}
n \\
m
\end{array}\right) p^{m}(1-p)^{n-m}
$$

Se puede comprobar que silos valores de $n$ y $m$ son grandes,el cálculo de $P_{n}(m)$ a partir de la aplicación directa de la fórmula (1.2) involucra técnicamente grandes dificultades (Gnedenko, [3, (1978)]).

A pesar de que en la actualidad, con el nivel de desarrollo que ha alcanzado el hardware de las computadoras, estos cálculos se pueden desarrollar en unos pocos minutos, siguen presentes los problemas de la naturaleza acotada de la representación de valores numéricos en ellas. Además, desde el punto de vista de la enseñanza de las probabilidades es necesario mostrar a los estudiantes como fueron evolucionando en la historia esos cálculos, en una época en la cual no existían medios de cómputo para efectuar los mismos.

La fórmula de Stirling puede ayudar a resolver el problema decalcularlos factoriales, pero no el del producto $p^{m}(1-p)^{n-m} p^{m} q^{n-m}$ (Linares; Martínez; Hernández, [6, (1983)]), surgiendo el problema de encontrar fórmulas aproximadas simples para el cálculo de las mismas con un cierto grado de exactitud.

Una fórmula de este tipo fue encontrada en 1730 por Abraham De Moivre para el caso particular de un esquema de Bernoulli con $p=q=0.5$, la que apareció por primera vez en la segunda edición de su libro "The Doctrine of Chances", publicado en 1738. La fórmula dada por De Moivre fue luego generalizada por Pierre-Simon Laplace en 1812 (Laplace, [5, (1812)]) para el caso de $p$ arbitrario, $0<p<1$, a 
partir de entonces conocida como Teorema local de De Moivre-Laplace (Gnedenko, [3, (1978)]).

\section{Teorema 1.1 (Teorema local de De Moivre-Laplace).}

Si la probabilidad de ocurrencia de un evento $A$ en $n$ repeticiones independientes de un experimento aleatorio $\xi$, es constante e igual a $p, 0<p<1$, entonces la probabilidad $P_{n}(m)$ de que en $n$ repeticiones del experimento aleatorio $\xi$ el evento $A$ ocurra exactamente $m$ veces, satisface la relación:

$$
\frac{\sqrt{n p q} \cdot P_{n}(m)}{\frac{1}{\sqrt{2 \pi}} e^{-\frac{1}{2} x^{2}}} \rightarrow 1 \text { cuando } n \rightarrow \infty, \quad \text { con } x=\frac{m-n p}{\sqrt{n p q}}
$$

La aproximación asintótica dada por De Moivre y Laplace va empeorando a medida que $p$ se aleja de 0.5 y para valores pequeños de $p$ ( menores que 0.1)la aproximación no es buena, incluso falla para valores de $p=0$. Sin embargo, debido a que existen problemas prácticos que requieren del cálculo de estas probabilidades para valores pequeños de $p$ (sucesos raros), surgió la necesidad de encontrar una fórmula asintótica especialmente adaptada a estas situaciones, obtenida por Siméon-Denis Poisson (Gnedenko, [3, (1978)]), la cual se cita a continuación:

\section{Teorema 1.2 (Teorema de Poisson)}

Si la probabilidad de ocurrencia de un evento $A$ en $n$ repeticiones independientes de un cierto experimento aleatorio $\xi$, es constante e igual a $p$, con $p$ o $q$ próximos a cero, entonces la probabilidad $P_{n}(m)$ de que en $n$ repeticiones el evento $A$ ocurra exactamente $m$ veces, satisface la relación:

$$
P_{n}(m) \approx \frac{e^{-\lambda} \lambda^{m}}{m !}, \quad \text { con } \lambda=n p
$$

Además de las situaciones presentadas en los teoremas anteriores se puede estar interesado en calcular, no la probabilidad de que el evento éxito ocurra exactamente $m$ veces en $n$ repeticiones de un ensayo de Bernoulli, sino que tome valores en un cierto rango $\left(m_{1}, m_{2}\right)$, con $0 \leq m_{1} \leq n$ y $0 \leq m_{2} \leq n$. La solución a este problema se expresa en el teorema integral de De Moivre-Laplace (Gnedenko, [3, (1978)]).

\section{Teorema 1.3 (Teorema integral de De Moivre-Laplace)}

Si $m$ es el número de ocurrencias de un evento $A$ en $n$ repeticiones independientes de un experimento aleatorio $\xi$, en cada una de las cuales la probabilidad de ocurrencia del evento $A$ es igual a $p, 0<p<1$, entonces se satisface la siguiente relación uniformemente en $a$ y $b$, con $-\infty \leq a<b \leq+\infty$

$$
P\left(a \leq \frac{m-n p}{\sqrt{n p q}}<b\right)-\frac{1}{\sqrt{2 \pi}} \int_{a}^{b} e^{-\frac{1}{2} x^{2}} d x \rightarrow 0 P\left(a \leq \frac{m-n p}{\sqrt{n p q}}<b\right)-\frac{1}{\sqrt{2 \pi}} \int_{a}^{b} e^{-\frac{1}{2} x^{2}} d x \rightarrow 0, \text { cuando } n \rightarrow \infty
$$


Como resultado de la aplicación del teorema integral de De Moivre-Laplace, se pueden resolver tres problemas que usualmente se presentan en la práctica (Gnedenko, [3, (1978)]), los cuales se describen a continuación:

a.) Determinar la probabilidad $\beta$ de que la frecuencia de ocurrencia de un evento $A$, considerado como el éxito, se desvíe de la probabilidad $p$, en valor absoluto, en no más de una cantidad $\alpha$, esto es, determinar el valor de $\beta$ que viene dado por la expresión $\beta=2 \Phi\left(\alpha \sqrt{\frac{n}{p(1-p)}}\right)-1$, donde

$$
\Phi(x)=\frac{1}{\sqrt{2 \pi}} \int_{-\infty}^{x} e^{-\frac{1}{2} x^{2}} d x
$$

b.) Determinar el número mínimo de repeticiones $(n)$ de un ensayo de Bernoulli que se debe realizar, para que, con una probabilidad no menor que $\beta$, la frecuencia de ocurrencia de un evento $A$ se desvíede la probabilidad $p$, en valor absoluto, en no más de $\alpha$, el cual viene dado por la expresión

$$
n=\left\lfloor\frac{p(1-p)}{\alpha^{2}}\left(\Phi^{-1}\left(\frac{1+\beta}{2}\right)\right)^{2}\right\rfloor+1
$$

c.) Dada una probabilidad $\beta$ y el número de repeticiones $n$ de un ensayo de Bernoulli, determinar el límite de las posibles variaciones, en valor absoluto, entre la frecuencia de ocurrencia de un evento $A,\left(\frac{m}{n}\right)\left(\frac{\mu}{n}\right)$, y su probabilidad de ocurrencia $p$, el cual viene dado por la expresión

$$
\alpha=\sqrt{\frac{p(1-p)}{n}} \Phi^{-1}\left(\frac{1+\beta}{2}\right)
$$

\subsection{Características de las funciones implementadas en el paquete ExpRep}

En la asignatura Teoría de las Probabilidades de la carrera de Licenciatura en Matemática de la Universidad de Oriente, en Santiago de Cuba, se enseña el tema "Repetición de experimentos", el cual incluye el estudio de los teoremas límites mencionados en el capítulo anterior.

Sobre la base de un análisis histórico-lógico de cómo ha venido enseñándose este tema en los diferentes planes de estudios, se ha podido observar que, al no incorporarse las demostraciones de estos teoremasen dichos planes por su nivel de complejidad, los estudiantes asumen estos de forma mecánica, sin estar conscientes de su validez y aplicabilidad. Tampoco resulta posible verificar numéricamente su validez dado que, para los valores para los cuales tienen sentido,la determinación de las probabilidades mediante la fórmula exacta (fórmula binomial) involucra cálculos voluminosos y potenciales problemas de precisión (Gmurman, [2, (1974)]), que impide comparar los resultados obtenidos al aplicar los teoremas límites y la fórmula exacta.

Todo loanterior evidencia la necesidad de contribuir a la comprensión y sistematización de esecontenido desde la dinámica del proceso de enseñanza-aprendizaje de la asignaturaTeoría de las Probabilidades. 
Debido a que el aprendizaje de los estudiantes está condicionado, entre otras cosas, por el grado de motivación que tengan por el contenido que se les enseña, el profesor debe realizar algunas acciones que despierten su interés por la información a recibir. En esta dirección es que se concibe el paquete ExpRep, el cual constituye un medio auxiliar para la enseñanza de los teoremas límites.

ExpReppermite calcular la probabilidad $P_{n}(m)$ de ocurrencia de un evento dado en un gran número de repeticiones de un experimento de tipo Bernoulli, para diferentes valores de $p$ (probabilidad de ocurrencia del evento en cada repetición delexperimento), así como realizar simulaciones que muestran analítica y gráficamente la veracidad de los teoremas límites, mediante el cálculo exacto de las probabilidades aplicando la fórmula binomial y la obtenida de forma aproximada al aplicar esos teoremas. Se muestran además los errores que se cometen en estas aproximaciones.

R es un potente lenguaje de programación para el cálculo estadístico y la exploración, análisis y visualización de datos. Es un lenguaje interpretado de código abierto, que ha tenido un fuerte y rápido crecimiento, contando con una comunidad muy activa de desarrolladores que han incorporado o mejorado una gran cantidad de paquetes que facilitan la solución de problemas en diversos campos del saber. Por todas estas bondades los autores de este trabajo seleccionaron el lenguaje Ren su versión 3.3.0, con la ayuda del entorno de desarrollo integrado (IDE) RStudio versión 0.99.896, para desarrollar ExpRep, y colateralmente contribuir a motivar a que los estudiantes incorporen dicho lenguaje en su formación profesional, mediante la elección de un curso optativo especialmente diseñado para el aprendizaje de R.

Las funciones implementadas dentro del paquete ExpRep, el cual fue incorporado al fondo de paquetes de la CRAN (ComprehensiveRArchive Network) con fecha 28 de junio de 2017 y cuyos códigos en $\mathrm{R}$ se pueden obtener en la dirección https://cran.r-project.org/web/packages/ExpRep/, se describen a continuación.

a.) Local_Theorem.

Dados $n$ ensayos de Bernoulli con probabilidad de éxito $p$, la función Local_ Theorem calcula y retorna la probabilidad aproximada de que un evento $A$, considerado como el éxito, ocurra exactamente $m$ veces, empleando el teorema local de De Moivre-Laplace. El encabezado de la función es:

Local_ Theorem <- function $(n, m, p)$

Un ejemplo de uso, en el cual el resultado de la corrida en R viene precedido de la notación [1], es:

$>$ Prob <- Local_ Theorem $(n=100, m=50, p=0.02)$

$>$ Prob

[1] $6.085801 \mathrm{e}-14$

El valor 6.085801e-14 representa el cálculo aproximado de $P_{100}(50)$, esto es,

$$
P_{100}(50) \approx 6.085801 \mathrm{e}-14=\frac{1}{\sqrt{100 \cdot 0.02 \cdot 0.98}} \frac{1}{\sqrt{2 \pi}} e^{-\frac{1}{2} x^{2}}, \text { donde } x=\frac{50-100 \cdot 0.02}{\sqrt{100 \cdot 0.02 \cdot 0.98}}
$$

b.) Poisson_Theorem. 
Dados $n$ ensayos de Bernoulli, con $n$ suficientemente grande (mayor que 50), con probabilidad de éxito $p$ pequeña (menor que 0.1 ), la función Poisson_Theorem calcula la probabilidad aproximada de que un evento $A$, considerado como el éxito, ocurra exactamente $m$ veces, basada en el teorema de Poisson. El encabezado de la función es:

Poisson_Theorem <- function $(n, m, p)$

Un ejemplo de uso es:

$>$ Prob <- Poisson_Theorem $(n=100, m=50, p=0.002)$

$>$ Prob

\section{[1] $3.030861 \mathrm{e}-100$}

El valor 3.030861e-100 representa el cálculo aproximado de $P_{100}(50)$, esto es,

$$
P_{100}(50) \approx \frac{e^{-\lambda} \lambda^{50}}{50 !}=3.030861 \mathrm{e}-100, \text { donde } \lambda=n p=0.2
$$

c.) Integral_Theorem.

Dados $n$ ensayos de Bernoulli con probabilidad de éxito $p$, la función Integral_Theorem calcula la probabilidad de que el número de ocurrencias de un evento $A$, considerado como el éxito, ocurra no menos de linf veces y no más de lsup veces. El encabezado de la función es:

Integral_Theorem $<-$ function $(n, p$, linf, lsup)

Un ejemplo de uso es:

$>$ Prob <- Integral_Theorem $(n=100, p=0.5$, linf $=5$, lsup=70)

tt $>$ Prob

[1] 0.9999683

donde 0.9999683 representa el cálculo aproximado de $P_{100}(5 \leq m \leq 70)$, o sea,

$$
\begin{aligned}
P(5 \leq m<70) & =P\left(\frac{5-100 \cdot 0.5}{\sqrt{100 \cdot 0.5 \cdot 0.5}} \leq \frac{m-100 \cdot 0.5}{\sqrt{100 \cdot 0.5 \cdot 0.5}}<\frac{70-100 \cdot 0.5}{\sqrt{100 \cdot 0.5 \cdot 0.5}}\right) \\
& \approx \frac{1}{\sqrt{2 \pi}} \int_{-9}^{4} e^{-\frac{1}{2} x^{2}} d x=0.9999683
\end{aligned}
$$

d.) ApplicIntegralTheo.

Esta función permite hacer los cálculos asociados a tres aplicaciones del teorema integral de De Moivre-Laplace. El encabezado de la función es:

ApplicIntegralTheo <- function(Applic, n, p, alpha, beta)

La función retorna un valor numérico que representa al valor de $n$, alpha o beta, en dependencia del valor que se le asigne al parámetro Applic("n", "alpha" o "beta" respectivamente). En 
la llamada de la función se omitirá de la lista de parámetros aquel que coincida con el valor asignado.

A continuación se muestran ejemplos de corridas de esta función, observar como en el primer ejemplo el parámetro Applictoma el valor "beta" y el parámetro beta no recibe ningún valor en la transferencia de parámetros o simplemente no se pone como parte de los argumentos en el llamado de la función:

> beta <- ApplicIntegralTheo(Applic ="beta", $\mathrm{n}=369, \mathrm{p}=0.4$, alpha=0.05)

$>$ beta

[1] 0.95006827

$$
0.95006827=2 \Phi\left(0.05 \cdot \sqrt{\frac{369}{0.4 \cdot 0.6}}\right)-1 .
$$

> alpha <- ApplicIntegralTheo (Applic="alpha", $n=369, p=0.4$, beta=0.95)

$>$ alpha

[1] 0.04998509

$$
\begin{aligned}
& 0.04998509=\sqrt{\frac{0.4 \cdot 0.6}{369}} \Phi^{-1}\left(\frac{1+0.95}{2}\right) . \\
& >n<- \text { ApplicIntegralTheo (Applic="n", } p=0.4, \text { alpha=0.05, beta=0.95) } \\
& >\mathrm{n}
\end{aligned}
$$

[1] 369

$$
369=\left\lfloor\frac{0.4 \cdot 0.6}{0.05^{2}}\left(\Phi^{-1}\left(\frac{1+0.95}{2}\right)\right)^{2}\right\rfloor+1 .
$$

\subsection{Simulaciones de los experimentos y su empleo en la enseñanza}

Como plantean Grima y Riba [4, (1995)], el creciente empleo de las computadoras personales y repositorios de software estadístico contribuyen a la utilización de la simulación como una herramienta importante en la enseñanza de la estadística y el cálculo de probabilidades.

Además de las funciones descritas en el capítulo anterior, dentro del paquete se implementan cuatro funciones que permiten realizar simulaciones, las tres primeras encaminadas a demostrar gráfica y analíticamente el planteamiento de los teoremas límites referidos, y la cuarta a mostrar la relación que existe entre la probabilidad de ocurrencia de un evento y la frecuencia con que este ocurre al repetirse muchas veces determinado fenómeno, relación descubierta por Bernoulli y que recibe el nombre de Teorema de Bernoulli. En lo que sigue se describen estas funciones, en cuyos encabezados aparecen los valores que por defecto se dieron a algunos de los parámetros, usados casi idénticamente en las llamadas. 
a.) S_Local_Limit_Theorem

Dados $n$ ensayos de Bernoulli, con probabilidad de éxito $p$, la función S_Local_Limit_Theorem calcula y grafica las probabilidades exactas y aproximadas de que el evento considerado como el éxito ocurra exactamente $m$ veces, con $0 \leq m \leq n$. Así mismo, la función calcula la diferencia entre esas probabilidades (Compare $=$ TRUE) y muestra todos los cálculos en una tabla (Table=TRUE) y/o mediante un gráfico con las probabilidades (Graph=TRUE) y/o con los errores (GraphE=TRUE). Su encabezado es:

S_Local_Limit_Theorem $\ll$ - function $(n, p$, Compare=TRUE, Table=TRUE, Graph=TRUE, GraphE=TRUE)

Por ejemplo, para la llamada:

< S_Local_Limit_Theorem $(n=170, p=0.5$, Compare=TRUE, Table=TRUE, Graph=TRUE, GraphE=TRUE)

Las salidas se muestras en la figura 1.1 y la tabla 1.1:

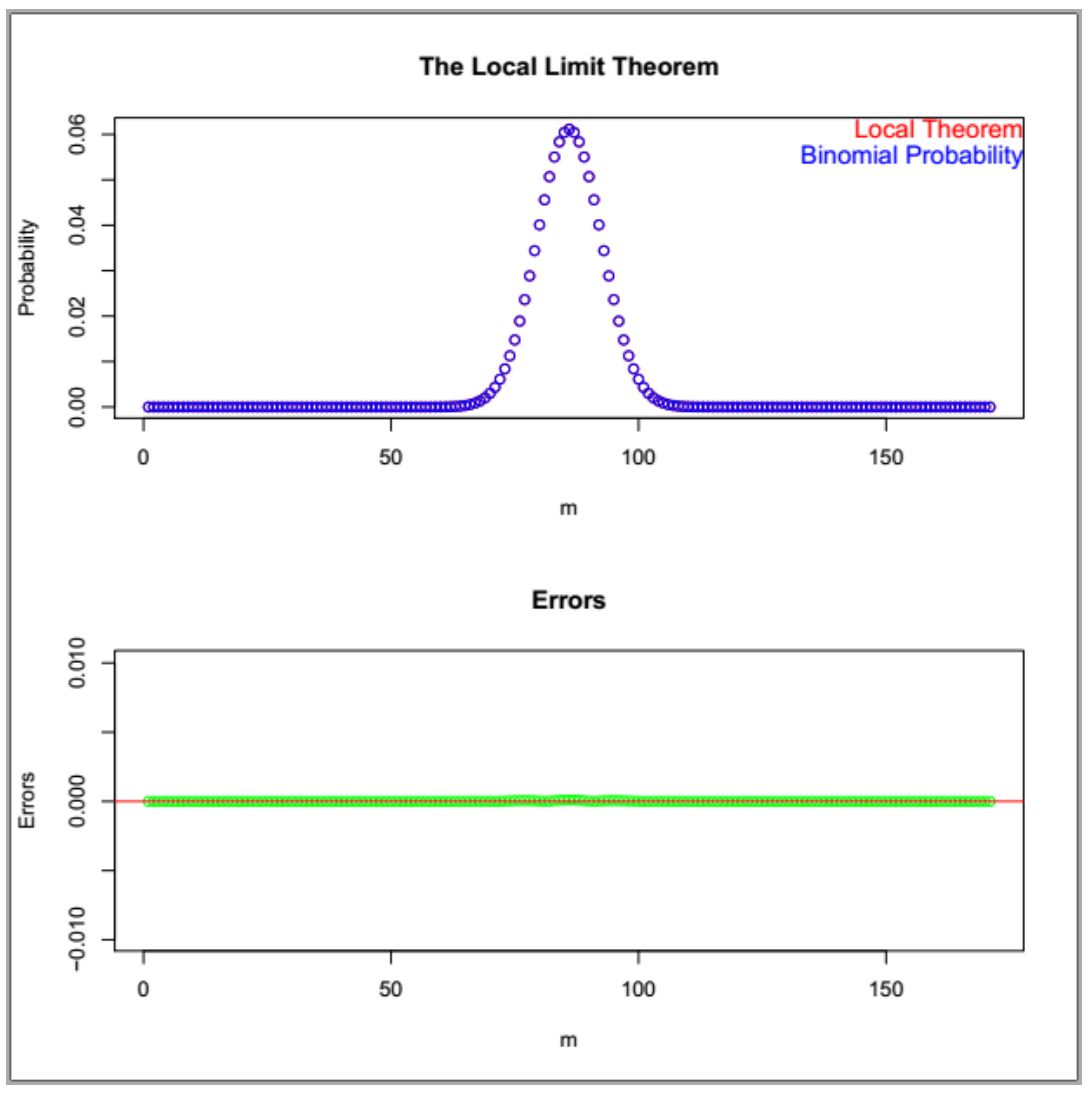

Figura 1.1: . Resultados numéricos de aplicar la fórmula exacta y teorema local de De Moivre-Laplace al cálculo de probabilidades para $n=170$ y $p=0.5$ 
Tabla 1.1: Probabilidades aplicando la fórmula exacta, el teorema local de De Moivre-Laplace y el de Poisson. Fuente: Elaboración propia.

\begin{tabular}{|l|l|l|l|l|}
\hline$m$ & $x$ & PBinomial & TLocal & Difference \\
\hline 0 & $-13,038405$ & $6,681912 \mathrm{e}-52$ & $7,441914 \mathrm{e}-39$ & $7,441914 \mathrm{e}-39$ \\
\hline 1 & $-12,885012$ & $1,135925 \mathrm{e}-49$ & $5,434558 \mathrm{e}-38$ & $5,434558 \mathrm{e}-38$ \\
\hline 2 & $-12,731619$ & $9,598566 \mathrm{e}-48$ & $3,876370 \mathrm{e}-37$ & $3,876370 \mathrm{e}-03$ \\
\hline$\ldots$ & $\ldots$ & $\ldots$ & $\ldots$ & $\ldots$ \\
\hline 168 & 12,731619 & $9,598566 \mathrm{e}-48$ & $3,876370 \mathrm{e}-37$ & $3,876370 \mathrm{e}-37$ \\
\hline 169 & 12,885012 & $1,135925 \mathrm{e}-49$ & $5,434558 \mathrm{e}-38$ & $5,434558 \mathrm{e}-38$ \\
\hline 170 & 13,038405 & $6,681912 \mathrm{e}-52$ & $7,441914 \mathrm{e}-39$ & $7,441914 \mathrm{e}-39$ \\
\hline
\end{tabular}

En la salida gráfica se puede apreciar la buena aproximación que se obtiene de las probabilidades aplicando la fórmula exacta (fórmula binomial, en azul) y el teorema local de De Moivre-Laplace (en rojo), por esta razón ambas gráficas se solapan, lo cual le permite al estudiante comprobar numéricamente la validez del teorema local de De Moivre-Laplace.

b.) S_Poisson_Theorem

Dados $n$ ensayos de Bernoulli, con probabilidad de éxito $p$, la función S_Poisson_Theorem calcula y muestra gráficamente, usando el teorema de Poisson, las probabilidades exacta y aproximada de que el evento considerado como el éxito ocurra exactamente $m$ veces, con $0 \leq m \leq n$. Además, la función calcula la diferencia entre esas probabilidades y muestra todos los cálculos en una tabla. Un posible encabezado es:

S_Poisson_Theorem <- function ( $n, p$, Compare=TRUE, Table=TRUE, Graph = TRUE, GraphE = TRUE)

en el cual los parámetros tienen igual significado que para la función S_Local_Limit_Theorem, con excepción de GraphE, que de ser TRUE muestra los gráficos de las diferencias entre las probabilidades calculadas bajo la fórmula binomial y el teorema de Poisson, y bajo la fórmula binomial y el teorema local, respectivamente.

Sea la llamada:

$<$ S_Poisson_Theorem $(\mathrm{n}=169, \mathrm{p}=0.002$, Compare=TRUE, Table=TRUE, Graph=TRUE, GraphE=TRUE)

las salidas se muestranen la figura 1.2 y la tabla 1.2, de la cual solo se presentan algunas filas: 

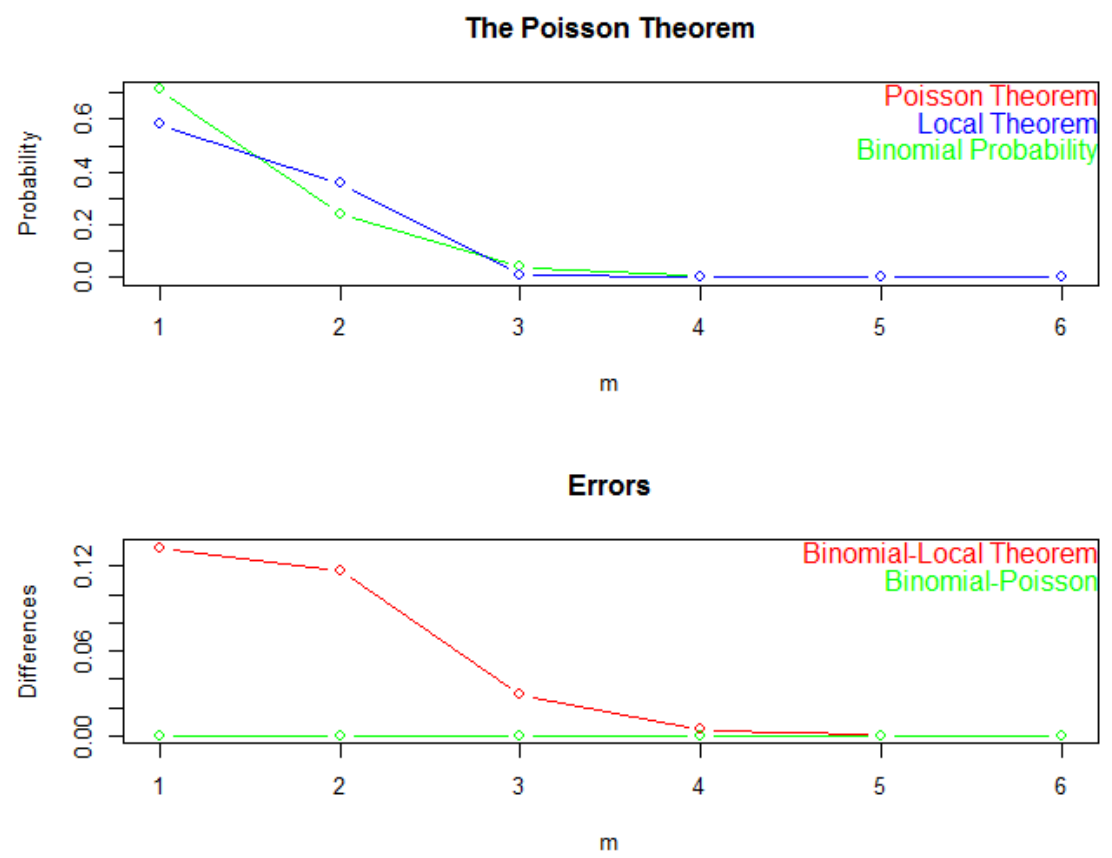

Figura 1.2: Tabulación de resultados mostrados en la gráfica 2

Tabla 1.2: Probabilidades aplicando la fórmula exacta y el teorema integral de De Moivre-Laplace, Fuente: Elaboración propia.

\begin{tabular}{|l|l|l|l|l|l|l|}
\hline$m$ & $x$ & PBinomial & TPoisson & Difference1 & TLocal & Difference2 \\
\hline 0 & $-0,5819599$ & 0,712953 & 0,713195 & 0,000242 & 0,579887 & 0,133066 \\
\hline 1 & 1,1398150 & 0,241461 & 0,241060 & 0,000401 & 0,358735 & 0,117274 \\
\hline 2 & 2,8615899 & 0,040646 & 0,000739 & 0,039907 & 0,011448 & 0,029198 \\
\hline$\ldots$ & $\ldots$ & $\ldots$ & $\ldots$ & $\ldots$ & $\ldots$ & $\ldots$ \\
\hline 167 & 286,9544529 & 0 & 0 & 0 & 0 & 0 \\
\hline 168 & 288,6762278 & 0 & 0 & 0 & 0 & 0 \\
\hline 169 & 290,3980027 & 0 & 0 & 0 & 0 & 0
\end{tabular}

Differencel es la diferencia (en valor absoluto) entre las probabilidades calculadas usando la fórmula exacta y el teorema de Poisson, y Difference2 es la diferencia (en valor absoluto) entre las probabilidades calculadas usando la fórmula exacta y el teorema local de De Moivre-Laplace.

En la figura 2 se muestran las probabilidades obtenidas al emplear el teorema local de De MoivreLaplace y el de Poisson, y la fórmula binomial (parte superior de la gráfica) y las diferencias o errores que se cometen al aplicar uno $\mathrm{u}$ otro teorema y la fórmula binomial (parte inferior de la figura), con el fin de que el estudiante pueda verificar la conveniencia de utilizar el teorema de Poisson cuando $p$ es muy pequeña en lugar del teorema local de De Moivre-Laplace. Con el objetivo de lograr una representación más clara, solo se muestran las probabilidades para aquellos valores de $m$ en los cuales los errores sean mayores que 0,0000005 .

Debido a la buena aproximación que se obtiene al aplicar la fórmula exacta (fórmula binomial, en verde) y el teorema de Poisson (en rojo) en la parte superior de la figura 1.2, las dos gráficas 
se solapan, sin embargo, en la gráfica de los errores se puede apreciar que para valores pequeños de $m(m \leq 4)$, al emplear el teorema de Poisson se comete un error más pequeño (color verde) que al aplicar el teorema local de De Moivre-Laplace (color rojo).

c.) S_Integral_Theorem

Dados $n$ ensayos de Bernoulli con probabilidad de éxitop, la función Integral_Theorem calcula y grafica las probabilidades exacta y aproximada de que el evento considerado como el éxito ocurra no menos de linf $+i$ veces y no más de lsup veces, con $0 \leq i \leq($ lsup $-l$ inf $)-1$. La función calcula también la diferencia entre esas probabilidades y muestra todos los cálculos en una tabla.Su encabezado es:

S_Integral_Theorem <- function(n, p, linf, lsup, Compare=TRUE, Table=TRUE, Graph=TRUE, GraphE=TRUE )

Los parámetros n, p, Compare, Table, Graph y GraphE tienen el mismo significado que los de igual nombre en la función S_Local_Limit_theorem. Para la llamada:

$<$ S_Integral_Theorem ( $n=200, p=0.5$, linf $=0$, lsup=150, Compare=TRUE, Table=FALSE, Graph=TRUE, GraphE=TRUE)

La salida será:
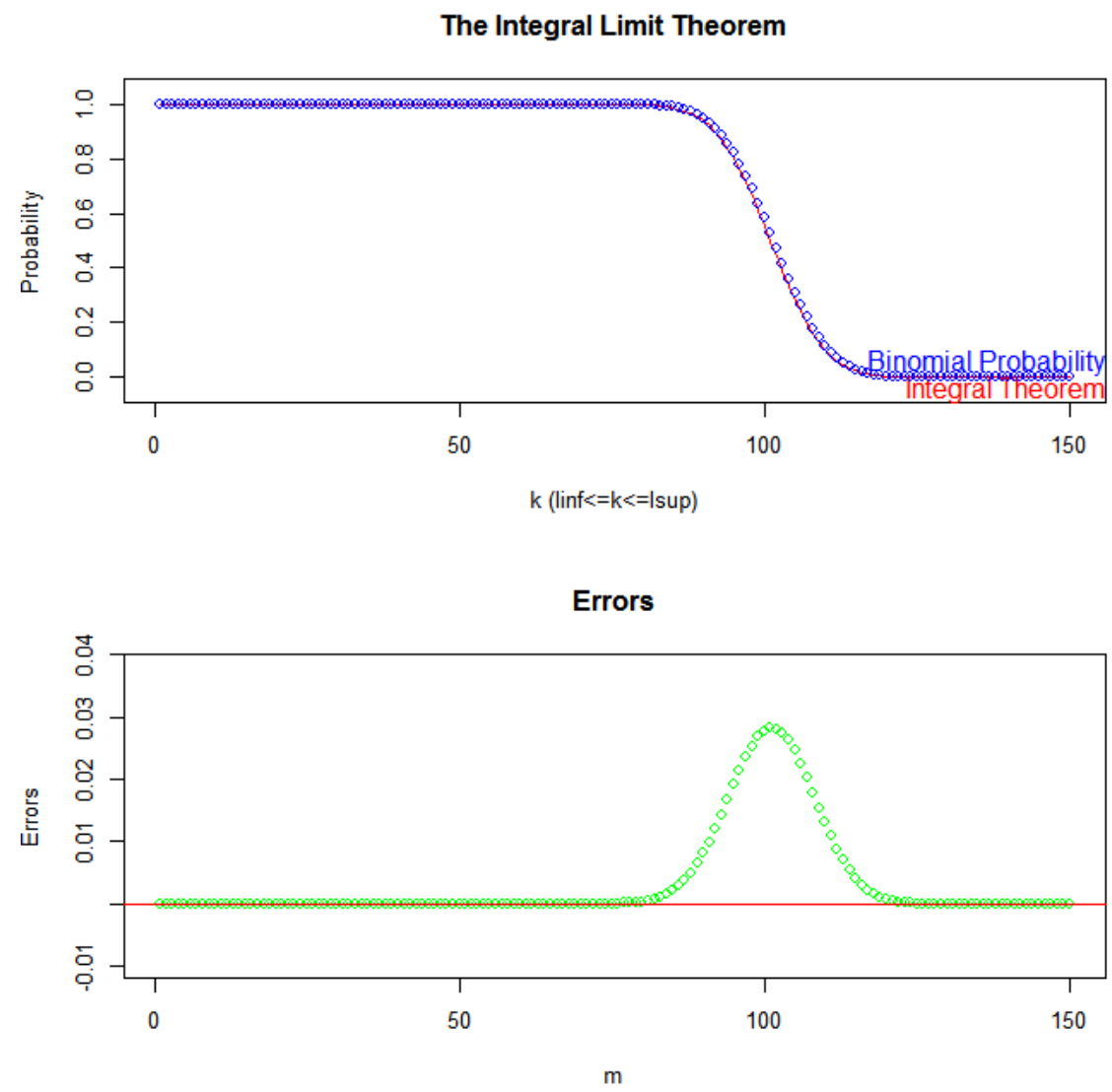

Figura 1.3: Simulación del experimento de Buffon para cantidad de muestras 100, 1000, 1500 y 6000 . Fuente: Elaboración propia. 
Al igual que en la primera figura, en la salida gráfica se puede apreciar la buena aproximación que se obtiene de las probabilidades aplicando la fórmula exacta (fórmula binomial, en azul) y el teorema integral de De Moivre- Laplace (en rojo).Se observa que el error de aproximación crece hasta que $m=n p$ y luego comienza a decrecer, con un valor máximo que es inferior a 0.03. En este caso la tabla no aparece pues al parámetro Table se le asignó el valor FALSE.

d.) Buffon

Esta función implementa una simulación de un experimento de Georges Louis Leclerc, Conde de Buffon, que consiste en lanzar una moneda un número suficientemente grande de veces y analizar la estabilidad de la frecuencia de ocurrencia del evento "aparición de cara", que es el definido como éxito (Linares; Martínez; Hernández, [6, 1983]). Su encabezado es:

Buffon $<-$ function ( $p$, width, $r$ )

donde $p$ es la probabilidad de ocurrencia de algún evento,width es el ancho de la banda gráfica en que se representan los valores de probabilidad y $r$ es un arreglo de cuatro valores, los cuales representan la cantidad de repeticiones del experimentoa realizar.

Como salida la función dibuja cuatro gráficos, cada uno de los cuales es una simulación del experimento de Buffon para cada cantidad de repeticiones contenida en el arreglo $r$.

Por ejemplo, para la llamada:

$<\operatorname{Buffon}(p=0.5$, width=0.2, $r=c(100,1000,1500,6000))$

se mostraría la figura 1.4:
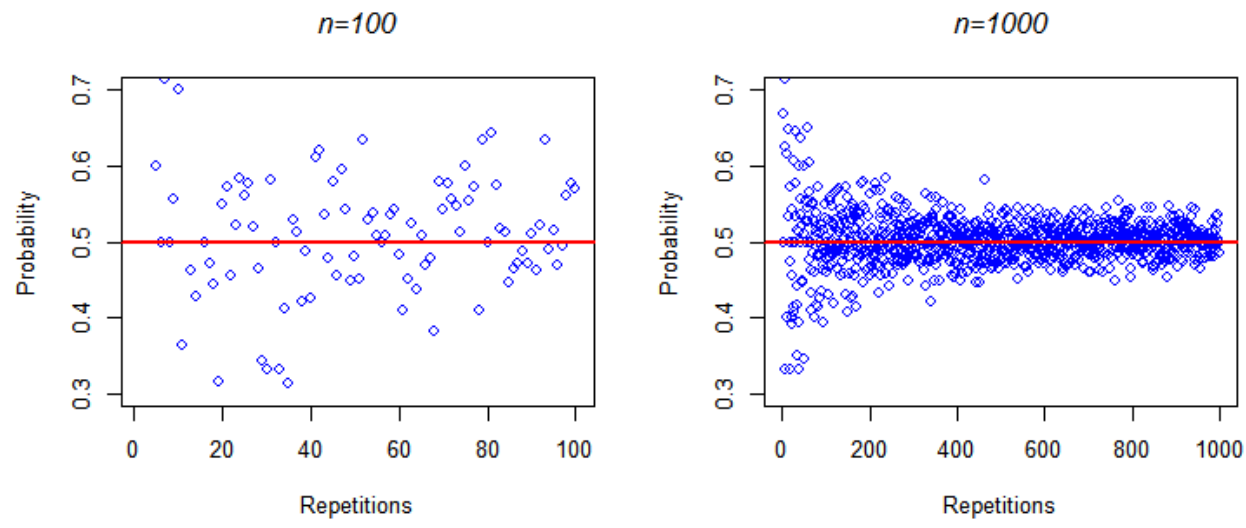

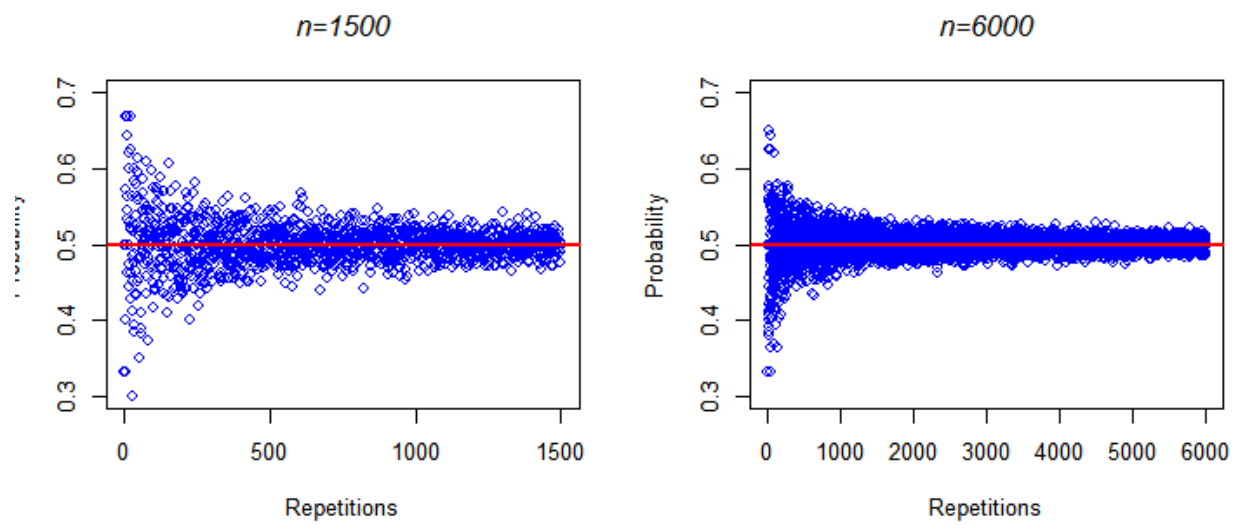

Figura 1.4: Simulación del experimento de Buffon para cantidad de muestras 100, 1000, 1500 y 6000 . Fuente: Elaboración propia

En la figura el estudiante puede observar como a medida que el número $n$ de repeticiones del experimento aumenta, la probabilidad de ocurrencia del evento tiende a aproximarse cada vez más a la línea roja que representa la probabilidad real de ocurrencia de dicho evento. Esto es empleado como recurso didáctico cuando se le enseña al estudiante la definición estadística de probabilidad, la cual se define a partir de la estabilidad de la frecuencia de ocurrencia de un evento en un gran número de repeticiones del experimento.

Tradicionalmente el tema de "Repetición de experimentos" se ha enseñado mediante una conferencia en laque seexplican los fundamentos teóricos del tema y los teoremas límites,y una clase práctica donde el estudiante de forma manual o con auxilio de tablas o de una calculadora le da solución a ejemplos de aplicación de la teoría recibida. La evaluación de este tema se realiza a través de un trabajo extraclase en el cual el estudiante da solución a los problemas planteados de forma similar a como lo hizo en la clase práctica.

En los cursos 2016-2017 y 2017-2018 se ha llevado a cabo un experimento didáctico con los estudiantes del segundo año de la carrera de Licenciatura en Matemática que reciben la asignatura Teoría de las Probabilidades, en el cual se enseña el tema de la forma tradicional, y se adiciona una práctica de laboratorio en la que los estudiantesdeben dar solución a problemas vistos en la clase práctica y otros adicionales. Este experimento permitió comprobar en ambos cursos una elevación del nivel de motivación en los estudiantes hacia el tema, que se evidenció en un número mayor de problemas resueltos, mayor precisión al seleccionar el teorema límite adecuado para cada situación planteada y resultados cualitativa y cuantitativamente superiores en el trabajo extraclase y en el examen final.

\subsection{Conclusiones}

En este artículo se ha presentado la funcionalidad del paquete ExpRep, elaborado en el lenguaje de programación $\mathrm{R}$, el cual permite calcular probabilidades aproximadas de eventos para determinadas 
situaciones que resultan difíciles de calcular aplicando las fórmulas exactas.En particular se ha programado el cálculo de la probabilidad de que un cierto evento, considerado como el éxito y cuya probabilidad de ocurrencia es $p$, ocurra un número determinado de veces o que el número de ocurrencias se encuentre en un cierto rango de valores, en un número grande de repeticiones de un ensayo de Bernoulli con probabilidad de éxito $p$.

Se presentan además cuatro funciones que permiten realizar simulaciones, lo que le da la posibilidad al estudiante de comprobar gráfica y numéricamente el cumplimiento de los teoremas local e integral de De Moivre-Laplace, Poisson y el teorema de Bernoulli, así como el experimento de Buffon.

A partir de los resultados obtenidos en el experimento didáctico aplicado a los estudiantes del segundo año de la carrera de Licenciatura en Matemática que reciben la asignatura Teoría de las Probabilidades, desde el curso 2016-2017, consistente en el empleo del paquete ExpRep en la docencia, se ha incrementado la atención de los estudiantes y su motivación por indagar un número mayor de situaciones que requieren de la aplicación de los teoremas límites, lo cual no se lograba con los métodos tradicionales de enseñanza de las probabilidades, por lo que se hace más efectiva la explicación del profesor y el aprendizaje de los estudiantes de los tópicos desarrollados en el paquete.

\section{Bibliografía}

[1] Gamboa, R. (2007). Uso de la tecnología en la enseñanza de las matemáticas. Cuadernos de investigación y formación en educación matemática, 2(3), 11-44.

[2] Gmurman, V. E. (1974). Teoría de las Probabilidades y Estadística Matemática. Moscú: Editorial Mir.

[3] Gnedenko,B.V. (1978).TheTheoryofProbability.Moscow: MirPublishers.

[4] Grima, P.; Riba, A. (1995). La simulación y la enseñanza de la estadística, casos prácticos. Estadística Española, 37(140), 409-434.

[5] Laplace, P.S. (1812).Théorie analytique des probabilités.Paris: Courcier.

[6] Linares, G.; Martínez, C.; Hernández, N. (1983).Probabilidades y Estadística. La Habana: Editorial Pueblo y Educación.

[7] Matloff, N. S. (2011).The art of R programming: tour of statistical software design.San Francisco: no starchPress.

[8] Olesker, L. (2015). Simulación: desafíos y oportunidades para la enseñanza de la probabilidad. Actas del CUREM 5,68-79.

[9] Osorio, M. A.; Suárez, A.; Uribe, C.C. (2013). Revisión de alternativas propuestas para mejorar el aprendizaje de la Probabilidad. Revista Virtual Universidad Católica del Norte, (38), 127-142. 